\title{
BMJ Open Waist-to-height ratio as a screening tool for cardiometabolic risk in children and adolescents: a nationwide cross- sectional study in China
}

\author{
Yalan Dou, ${ }^{1}$ Yuan Jiang, ${ }^{1}$ Yinkun Yan, ${ }^{2}$ Hongyan Chen, ${ }^{1}$ Yi Zhang, ${ }^{1}$ Xiaotian Chen, ${ }^{1}$ \\ Yin Wang, ${ }^{1}$ Hong Cheng, ${ }^{3}$ Xiaoyuan Zhao, ${ }^{3}$ Dongqing Hou, ${ }^{3}$ Jie Mi, ${ }^{2,3}$ Weili Yan (D) ${ }^{1}$
}

To cite: Dou Y, Jiang $Y$, Yan Y, et al. Waist-to-height ratio as a screening tool for cardiometabolic risk in children and adolescents: a nationwide cross-sectional study in China. BMJ Open 2020;10:e037040. doi:10.1136/ bmjopen-2020-037040

- Prepublication history for this paper is available online. To view these files, please visit the journal online (http://dx.doi org/10.1136/bmjopen-2020037040).

YD, YJ and YY are joint first authors.

Received 16 January 2020 Revised 11 February 2020 Accepted 23 May 2020

Check for updates

(c) Author(s) (or their employer(s)) 2020. Re-use permitted under CC BY-NC. No commercial re-use. See rights and permissions. Published by BMJ.

For numbered affiliations see end of article.

\section{Correspondence to}

Weili Yan;

yanwl@fudan.edu.cn and

Jie Mi;

jiemi12@vip.sina.com

\section{ABSTRACT}

Objectives To demonstrate the accuracy and flexibility of using waist-to-height ratio (WHtR) as a screening tool for identifying children and adolescents with cardiometabolic risk (CMR) across a wide range of prevalence levels among general paediatric populations.

Design A nationwide population-based cross-sectional study with all data collected at school settings in six cities of China.

Participants A total of 8130 children and adolescents aged 7-18 years with complete anthropometric and CMR measurements based on blood tests were recruited. Outcome measures Elevated blood pressure, dyslipidaemia, elevated fasting blood glucose and central obesity were measured. The primary outcome, CMRs, was defined as meeting three or more of the above risk factors The accuracy of WHtR for identifying CMRs was evaluated using areas under the curves (AUCs) with $95 \% \mathrm{Cl}$ of the receiver operating characteristic curve. The predictability of WHtR at given CMRs prevalence levels was estimated by positive predictive value (PPV) and negative predictive value.

Results Overall, $6.1 \%$ of study participants were presented with CMRs. WHtR had high AUCs ranging from $0.84(95 \% \mathrm{Cl} 0.81$ to 0.88$)$ to $0.88(95 \% \mathrm{Cl} 0.86$ to 0.90$)$ in the total population and age-subgroup and gendersubgroup. The overall optimal WHtR cut-off value was 0.467 , with boys having a higher cut-off than girls $(0.481$ vs 0.456 ). WHtR achieved an overall sensitivity of 0.89 and PPV of $18.8 \%$ at a specificity of 0.75 . The screening performance of WHtR remained satisfactory across a wide range of given CMRs prevalence levels $(5 \%, 10 \%$ and 20\%).

Conclusion WHtR as a screening tool could accurately and flexibly identify children affected with the clusters of three or more of CMR factors from the general paediatric population with various CMR prevalence levels. Our findings provide support for policy-making on early CMR identification and management in the high-risk group of children.

\section{INTRODUCTION}

Obesity is a growing global epidemic, with more than 340 million children and adolescents aged 5-19 being overweight or obese
Strengths and limitations of this study

- As the largest nationwide cross-sectional study in the paediatric population, our findings enhanced the generalisability of waist-to-height ratio (WHtR) to practice.

- The findings were based on objectively measured cardiometabolic risk (CMR) outcomes and waist circumstance in the large population.

- The large sample size allowed us to report optimal WHtR cut-off values to identify CMR for the entire population and age-specific and gender-specific subgroups, and simulate the predictability at a wide range of given CMR prevalence levels.

- Our analysis was limited to children with complete data on blood tests, which may cause selection bias in the research findings.

- Potential covariates, including the age of puberty, family history of cardiovascular disease, and obesity were not determined.

in 2016. ${ }^{1}$ Obesity in childhood is associated with multiple cardiometabolic risk (CMR) factors including hyperglycaemias, dyslipidaemia and hypertension, which predicts increased risk of obesity and cardiovascular diseases in adulthood. ${ }^{2}$ Early CMR identification in general paediatric population could improve the awareness of the self-risk status and further promote healthy lifestyles, which may potentially prevent the long-term risk of cardiovascular diseases. ${ }^{3}$ Given that blood tests screening CMR in asymptomatic children is unacceptable in routine health examinations, anthropometric indexes with good correlation with CMR in children population has the potential to be used for screening in practice. ${ }^{4-7}$

Previous work has demonstrated the promise of waist-to-height ratio (WHtR), an obesity-related anthropometric index, as an efficient tool for quick and mass screening of CMR in children. ${ }^{8-13}$ However, given the 
vast variability in CMR prevalence across populations, the performance of WHtR in detecting CMR or clusters of CMR at different prevalence levels remains unclear. The objective of the analysis reported herein was to confirm the accuracy and predictive value of WHtR screening for CMR in paediatric populations with diverse CMR prevalence levels for the first time, using a larger sample of children and adolescents in China.

\section{METHODS}

\section{Study population}

This was a multicentre cross-sectional study conducted from 1 January 2013 to 31 December 2015, which included six large cities in the northern (Beijing, Jinan and Changchun) and southern regions (Shanghai, Chongqing and Chengdu), as part of the China Child and Adolescent Cardiovascular Health study. ${ }^{9}$ Details of the study design and sampling methods have been reported elsewhere. ${ }^{14}$ Briefly, the stratified cluster random sampling method was used to select more than 100 children ( 50 boys and 50 girls) from each age group in each city to ensure the representativeness of subjects. A total of 8794 students from selected schools participated in relevant examinations, including anthropometric and blood pressure (BP) measurements, and blood samples collections. We excluded 664 participants who were younger than 7 or older than 18 years. Finally, 8130 children ( $53.2 \%$ male) were included in the analysis.

\section{Anthropometric measurements}

Anthropometric measurements including body weight, height and waist circumference (WC) were examined by standard protocols and instruments, where subjects were lightly clothed and barefoot. The WHtR was calculated as the WC in centimetres divided by the height in centimetres. Detailed measurement methods were previously reported..$^{914}$

\section{CMR factors}

CMR factors included elevated BP, dyslipidaemia, elevated fasting blood glucose (FBG) and central obesity. The study outcome, CMRs, was defined as meeting three or more of the above four factors. BP was obtained on three different days, with three times measurements during each visit and at least 2 weeks between visits. Seated BP was measured on the right arm by the HEM-7012 electronic sphygmomanometer (OMRON Corp, Kyoto, Japan). Elevated BP was defined as over 90th percentile for age-specific and gender-specific reference. ${ }^{15}$ Blood samples were collected after a 10 hours overnight fast. Dyslipidaemia was defined as having any of the following conditions: (1) triglycerides of $\geq 1.76 \mathrm{mmol} / \mathrm{L}$, (2) total cholesterol of $\geq 5.2 \mathrm{mmol} / \mathrm{L}$, (3) low-density lipoprotein cholesterol $\geq 3.38 \mathrm{mmol} / \mathrm{L}$ or (4) high-density lipoprotein cholesterol of $\leq 1.04 \mathrm{mmol} / \mathrm{L} .{ }^{16}$ Elevated FBG was defined as a value of $\geq 5.6 \mathrm{mmol} / \mathrm{L} .{ }^{17}$ Central obesity was defined using the age-specific and gender-specific WC cut-offs recommendation obesity. ${ }^{18}$

\section{Statistical analysis}

Demographic characteristics and body measurements were summarised as means and SD for continuous variables and absolute numbers and percentages for categorical variables. Body mass index (BMI) and WC were standardised by age and gender based on reference curves for Chinese children and adolescents, respectively. ${ }^{19}{ }^{20}$ Areas under the curves (AUCs) with $95 \%$ CI of the receiver operating characteristic (ROC) were used to measure the accuracy of WHtR for discriminating children with CMRs from the general paediatric population. Considering the critical role of sensitivity in screening CMRs, we believe that specificity can be compromised for a higher sensitivity within an acceptable extent when determining the optimal cut-off values. Accordingly, we determined the optimal cut-off values as a maximum sensitivity with a specificity of $\geq 0.75$ for different groups. This value is arbitrary selected for illustrative purposes alone. By using diagnostic tests, we predicted the screening performances, including sensitivity, specificity, positive predictive value (PPV) and negative predictive value (NPV), in the overall study population and agespecific and gender-specific subgroups. The performance of WHtR was also evaluated using the well-known cut-off of $0.5 .{ }^{21}$ Importantly, we reported PPV and NPV in a series of CMRs prevalence levels $(5 \%, 10 \%$ and $20 \%$ ) to validate the robustness of our results. All analyses were performed using Stata V.15.0.

\section{Patient and public involvement}

There was no direct public or patient involvement in the design and implementation of this survey.

\section{RESULTS}

The general characteristics of subjects are presented by geographic region and city in table 1 . Among the 8130 subjects, $53.2 \%$ were male and $55.9 \%$ were 12 years or younger. The overall levels of two obesity measures, BMI and $\mathrm{WC}$, were mildly above the national reference levels (0.6 SD for BMI, 0.2 SD for WC). The mean WHtR was $0.444 \pm 0.061$, with higher values in the northern region cities. The overall prevalence of CMRs was $6.1 \%$ in the entire population and ranged from $1.6 \%$ to $12.6 \%$ across cities, which was generally higher in the northern region than in the southern region.

WHtR showed consistent and satisfactory performances in identifying children with CMRs (table 2). The AUCs ranged from 0.84 (95\% CI 0.81 to 0.88 ) to $0.88(95 \%$ CI 0.86 to 0.90 ) in the total population as well as in age-specific and gender-specific subgroups. The overall optimal cut-off value of WHtR was 0.467 , and $28.4 \%$ of the study population having WHtR values above this cutoff. The prevalence of CMRs was 7.7\% in boys and $4.3 \%$ in girls, and the optimal WHtR cut-offs were 0.481 and 
Table 1 General characteristics of the study children

\begin{tabular}{|c|c|c|c|c|c|c|c|}
\hline \multirow[b]{2}{*}{ Measurements } & \multirow[b]{2}{*}{ Overall } & \multicolumn{3}{|c|}{ Northern region } & \multicolumn{3}{|c|}{ Southern region } \\
\hline & & Beijing & Jinan & Changchun & Shanghai & Chongqing & Chengdu \\
\hline n (\%) & $8130(100.0)$ & 1307 (16.1) & 1210 (14.9) & $1518(18.7)$ & 1621 (19.9) & $1143(14.1)$ & $1331(16.4)$ \\
\hline Age, year (mean $\pm S D)$ & $12.0 \pm 3.3$ & $11.2 \pm 2.8$ & $11.4 \pm 3.4$ & $12.0 \pm 3.4$ & $13.3 \pm 3.3$ & $10.5 \pm 2.6$ & $12.8 \pm 3.5$ \\
\hline Age $\leq 12$ years, $n(\%)$ & 4547 (55.9) & $854(65.3)$ & $758(62.6)$ & 854 (65.3) & 593 (36.6) & $873(76.4)$ & $613(46.1)$ \\
\hline Height (cm) & $151.7 \pm 16.5$ & $152.9 \pm 16.4$ & $150.3 \pm 17.4$ & $153.6 \pm 17.5$ & $154.9 \pm 15.4$ & $143.4 \pm 13.6$ & $153.1 \pm 15.4$ \\
\hline BMI $\left(\mathrm{kg} / \mathrm{m}^{2}\right)$ & $20.0 \pm 4.5$ & $19.9 \pm 4.4$ & $20.9 \pm 5.3$ & $21.2 \pm 5.3$ & $20.0 \pm 4.0$ & $18.7 \pm 3.4$ & $19.0 \pm 3.7$ \\
\hline BMI SDS & $0.6 \pm 1.2$ & $0.7 \pm 1.2$ & $1.0 \pm 1.3$ & $0.9 \pm 1.4$ & $0.4 \pm 1.2$ & $0.6 \pm 1.1$ & $0.2 \pm 1.1$ \\
\hline WC (cm) & $67.4 \pm 12.1$ & $69.4 \pm 12.6$ & $68.8 \pm 13.9$ & $69.4 \pm 13.5$ & $68.1 \pm 10.8$ & $62.3 \pm 9.4$ & $65.3 \pm 9.9$ \\
\hline WC SDS & $0.2 \pm 1.2$ & $0.5 \pm 1.1$ & $0.4 \pm 1.3$ & $0.3 \pm 1.3$ & $0.1 \pm 1.1$ & $-0.2 \pm 1.1$ & $-0.2 \pm 1.1$ \\
\hline
\end{tabular}

BMI, body mass index; CMRs, meeting three or more cardiometabolic risk factors; SDS, SD score; WC, waist circumference; WHtR, waist-toheight ratio.

0.456, respectively. In addition, the optimal WHtR cut-offs differed between two age groups by gender. In boys, the optimal WHtR cut-off was 0.487 for the group of age $\leq 12$ years with CMRs prevalence of $6.3 \%$, and was 0.468 for the group of age $>12$ years with CMRs prevalence of $9.4 \%$, while the optimal cut-offs for girls were stable at 0.456 for the two age groups with CMRs prevalence being $4.5 \%$ and $4.0 \%$, respectively. Overall, WHtR achieved sensitivities of 0.85 or over in identifying CMRs in the total population and age-specific and gender-specific subgroups at our chosen specificity of 0.75 or more. Intriguingly, using the well-reported WHtR cut-off of 0.5 , from the common sense of 'keep your waist below half of your height' for the adult population, the sensitivity was only 0.68 with a specificity of 0.86 .
To further demonstrate the application of $\mathrm{WHtR}$, we simulated its predictive performance at three given CMRs prevalence levels $(5 \%, 10 \%$ and $20 \%$ ) in table 3. As expected, the predictive ability of WHtR using proposed optimal cut-off values varied among age and gender subgroups, and increased with the given prevalence of CMRs. In the overall study population, the PPV was $15.9 \%$ at the CMRs prevalence of $5 \%$, and would achieve $47.3 \%$ at a prevalence of $20 \%$. Results in the subgroups analysis by age and gender showed similar trends. At a given prevalence of 5\%, PPVs were $15.4 \%$ for boys and $16.4 \%$ for girls, ranging from $15.5 \%$ to $16.6 \%$ across the age subgroups; for the prevalence of $20 \%$, PPVs reached $46.3 \%$ for boys and $48.3 \%$ for girls, ranging from $46.5 \%$ to $48.6 \%$ across all the age subgroups.

Table 2 Optimal cut-offs and performance of WHtR screening for CMRs among general paediatric population

\begin{tabular}{|c|c|c|c|c|c|c|c|c|c|}
\hline Group & $\mathbf{N}$ & Prevalence (\%) & AUC & $95 \% \mathrm{Cl}$ & Optimal cut-offs & Sensitivity & Specificity & PPV (\%) & NPV (\%) \\
\hline Total & 8130 & 6.1 & 0.87 & (0.85 to 0.88$)$ & 0.467 & 0.89 & 0.75 & 18.8 & 99.0 \\
\hline \multicolumn{10}{|l|}{ Gender } \\
\hline Boys & 4325 & 7.7 & 0.86 & (0.85 to 0.88$)$ & 0.481 & 0.88 & 0.75 & 22.2 & 98.6 \\
\hline Girls & 3805 & 4.3 & 0.84 & (0.81 to 0.88$)$ & 0.456 & 0.85 & 0.77 & 14.2 & 99.2 \\
\hline \multicolumn{10}{|l|}{ Age group } \\
\hline Age $\leq 12$ years & 4547 & 5.5 & 0.85 & (0.83 to 0.88$)$ & 0.471 & 0.88 & 0.75 & 16.7 & 99.0 \\
\hline Boys & 2444 & 6.3 & 0.87 & (0.84 to 0.89$)$ & 0.487 & 0.92 & 0.75 & 19.5 & 99.2 \\
\hline Girls & 2103 & 4.5 & 0.84 & (0.79 to 0.88$)$ & 0.456 & 0.85 & 0.77 & 14.7 & 99.1 \\
\hline Age $>12$ years & 3583 & 6.8 & 0.88 & (0.86 to 0.90$)$ & 0.458 & 0.92 & 0.75 & 20.9 & 99.2 \\
\hline Boys & 1881 & 9.4 & 0.87 & (0.85 to 0.89$)$ & 0.468 & 0.91 & 0.75 & 27.0 & 98.7 \\
\hline Girls & 1702 & 4.0 & 0.86 & (0.80 to 0.91$)$ & 0.456 & 0.85 & 0.78 & 13.6 & 99.2 \\
\hline
\end{tabular}

AUC, area under receiver operating characteristic curve; CMRs, meeting three or more cardiometabolic risk factors; NPV, negative predictive value; PPV, positive predictive value; WHtR, waist-to-height ratio. 
Table 3 Predictive performance of WHtR screening for CMRs at given prevalence levels

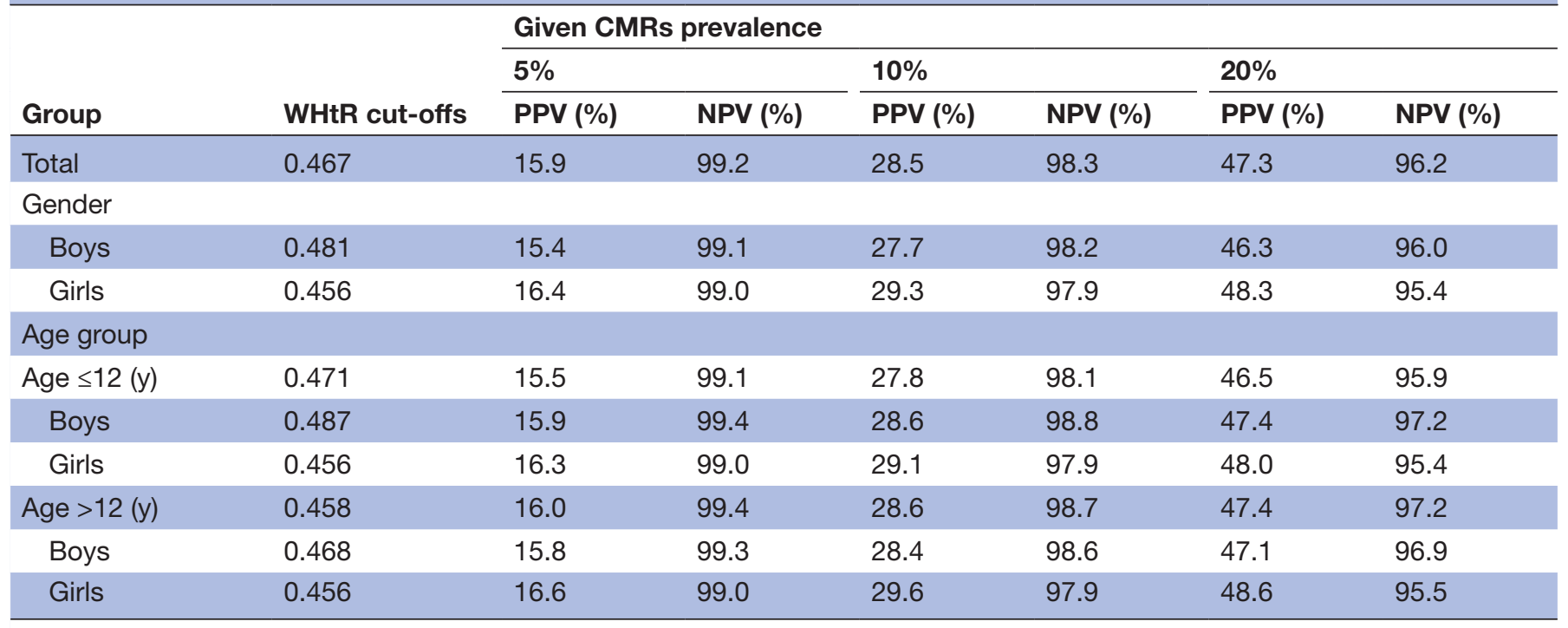

CMRs, meeting three or more cardiometabolic risk factors; NPV, negative predictive value; PPV, positive predictive value; WHtR, waist-toheight ratio.

\section{DISCUSSION}

Based on a large multicentre study population, we provided evidence to demonstrate the accuracy and flexibility of WHtR with appropriate cut-offs, in identifying the high-risk group affected by the clustering of three or more CMR factors from an apparently healthy population. The overall AUC reached 0.87 and remained consistent performance in gender-specific and age-specific subgroups. Importantly, these results were robust against various given CMRs prevalence levels, which were in line with previous large studies among European and American children. ${ }^{22-24}$ Furthermore, the optimal WHtR cutoff value of 0.467 was more accurate in discriminating CMRs in children population than the widely recommended cut-off of $0.50 .^{21}$

We found that different cut-off values of WHtR were required to achieve satisfactory discriminating performance across populations. In the HEALTHY study involving 6097 adolescents aged 10-13, a cut-off value of 0.52 showed a sensitivity of 0.80 and a specificity of 0.65 in identifying adolescents with clustered CMR. ${ }^{4}$ In 2935 Korean children and adolescents aged 10-19 years, a cut-off value of 0.491 showed a sensitivity over 0.96 and a specificity of $0.89 .^{25}$ In our study with a larger study sample covering a broad age span, the optimal cut-off of 0.467 of WHtR achieved a sensitivity of 0.89 and a specificity of 0.75 , which was acceptable for screening CMR from a general population for a public health purpose. Studies showed gender difference in WHtR but it was age-independent compared with other obesity-related indexes; ${ }^{92627}$ however, we found that the CMR could be different between the younger and the older children. ${ }^{9}$ In order to achieve better accuracy performance, we sought to find out the best cut-offs of WHtR for age and gender subgroups. Interestingly, we found that the optimal
WHtR cut-off values were slightly different in all the boys and in the two age groups, while were stable at 0.456 for girls. Nonetheless, the optimal cut-off values we identified gave a specificity of $>0.75$ and a sensitivity of $>0.85$ for the entire population and age and gender subgroups. We used 12 years old as the cut-off age for subgroup analysis in both genders, since it was a proposed time of transition from childhood to adolescence when secondary sexual characteristics appeared.

Our study also focused on the PPV parameter, the proportion of the true positives among screening-positive individuals, which was relevant to cost-effective considerations of using WHtR in practice. Besides the results based on the observed actual prevalence of CMRs, we also simulated PPVs of WHtR at a wider range of given prevalence levels. The PPV was $18.8 \%$ for the entire population with CMRs prevalence of $6.1 \%$, indicating that, approximately one in five children with WHtR $>0.467$ was affected by three or more CMR factors. In addition, WHtR also showed very high NPV of $98 \%$, suggesting that children with WHtR below the cut-off value were not likely affected by CMRs. These performances were better than those in the HEALTHY study with similar population CMR prevalence, in which the PPV was $12.3 \%$ at a cut-off of $0.52 .{ }^{4}$ WHtR could be more cost-effective in a population with higher CMR prevalence. In our simulation analysis, PPV raised to $47.3 \%$ at the given CMRs prevalence of $20 \%$. These findings could provide a cost-effective reference for policy-makers to promote appropriate screening strategies for CMR among different paediatric populations.

Blood tests were recently recommended to universal screening dyslipidaemia or elevated FBG in children aged 9-11 years to reduce CMR, ${ }^{28}$ however, widespread blood tests screening in asymptomatic individuals, was costly, time-consuming and even unacceptable in most 
routine health examinations. ${ }^{28}{ }^{29}$ In this context, WHtR index as an integral part of the regular healthcare could make the screening more rapidly and efficiently, as blood tests could be restricted to those children with WHtR over the cut-off values. For instance, given a CMRs prevalence of $6 \%$ in our study, rather than screening 100 children with blood tests to detect the six with three or more CMR factors, only 28 children with WHtR over the cut-offs of 0.467 need further blood tests to correctly identify the affected individuals. This also meant that the remaining nearly two-thirds of children could avoid the invasive blood tests, with $98 \%$ of them likely to be true metabolic healthy. WHtR could thus be promoted as a large-scale screening tool for children in routine health examinations for its efficiency and acceptability, which helped to improve awareness of the self-risk status and CMR reduction. Our findings may support the evidence-based guideline establishment and policy-making on WHtR screening for paediatric CMR, helping public health practitioners to improve primary prevention of cardiovascular disease later in adult life.

The current study had several strengths. First of all, this was the latest and largest nationwide study using WHtR to screen CMR in the paediatric population in China. The large sample size enhanced the generalisability of our findings, allowing us to identify the best WHtR cut-offs for age and gender subgroups. In addition, the findings were based on objectively measured CMR outcomes and WC, which were reliable. There were still some limitations. Of the China Child and Adolescent Cardiovascular Health study, our analysis only included 8130 children aged 7-18 who had blood tests, which may cause selection bias in the research findings. Our findings based on simulated analyses at given prevalence levels of CMRs need further validation. Moreover, the effects of potential covariates, including the age of puberty, family history of cardiovascular disease and obesity were not determined.

\section{CONCLUSIONS}

Our study provides strong evidence for the application of WHtR as an accurate and flexible tool in identifying the high-risk group with CMR in the general paediatric population. The screening performance of WHtR is satisfactory at given CMR prevalence levels. Our findings have important public health implications for evidencebased policy-making to promote CMR identification and management, which could be valuable in the primary prevention of cardiovascular disease later in adult life.

\footnotetext{
Author affiliations

${ }^{1}$ Department of Clinical Epidemiology, Children's Hospital of Fudan University, National Children's Medical Center, Shanghai, China

${ }^{2}$ Department of Non-Communicable Disease Management, Beijing Children's Hospital, Capital Medical University, National Center for Children's Health, Beijing, China

${ }^{3}$ Department of Epidemiology, Capital Institute of Pediatrics, Beijing, China
}

Acknowledgements We would like to thank the schools, students and research staff from Beijing, Shanghai, Chongqing, Chengdu, Jinan and Changchun who took part in the study. China Child and Adolescent Cardiovascular Health study research group: Jie Mi, MD, PhD, YinkunYan, MD, PhD, Hongbo Dong, MD, PhD, Department of Noncommunicable Disease Management, Beijing Children's Hospital, Capital Medical University, National Center for Children's Health, Beijing, China; Junting Liu, MS, Xiaoyuan Zhao, MS, Hong Cheng, MS, Dongqing Hou, MS, Fangfang Chen, MS, Guimin Huang, MS, Linghui Meng, PhD, Qin Liu, MS, Meixian Zhang, PhD, Wenpeng Wang, MS, Lijun Wu, PhD, Xinying Shan, MS, Ping Yang, MS, Department of Epidemiology, Capital Institute of Pediatrics, Beijing, China; Jinghui Sun, MD, Xiaona Wang, MD, Pediatric Cardiovascular Department, The First Hospital of Jilin University, Changchun, Jilin, China; Ying Li, MD, Department of Pediatrics, People's Hospital of Tonghua, Tonghua, Jilin, China; Weili Yan, PhD, Fang Liu, PhD, Yi Zhang, MPH, Yi Cheng, MS, Shaoke Chen, MS, Qian Zhao, MS, Fang Cao, MS, Kai Mu, MS, Dayan Niu, MS, Department of Clinical Epidemiology, Children's Hospital of Fudan University, Shanghai, China; Bo Xi, PhD, Min Zhao, PhD, Department of Epidemiology, School of Public Health, Shandong University, Jinan, Shandong, China; Feng Xiong, MD, Gaohui Zhu, PhD, Department of Endocrinology, Children's Hospital of Chongqing Medical University, Chongqing, China; Wenqing Ding, PhD, Ling Zhang, PhD, Leina Jia, MD, Department of Health Education and Child Health Care, School of Public Health, Ningxia University, Ningxia, China.

Contributors YD, YJ and YY are joint first authors. JM and WY are joint corresponding authors. JM, WY and YJ obtained funding. WY and JM designed the study. YY, YZ, $\mathrm{H}-\mathrm{yC}, \mathrm{XZ}$ and $\mathrm{DH}$ were involved in the implementation of the national study. YY, $\mathrm{H}-\mathrm{yC}, \mathrm{XC}$ and $\mathrm{YW}$ were involved in data cleaning and verification. $\mathrm{YD}, \mathrm{YJ}$ and $\mathrm{YY}$ analysed the data and drafted the manuscript. WY, JM and $\mathrm{H}-\mathrm{yC}$ contributed to the critical revision of the manuscript for important intellectual content. WY and $\mathrm{JM}$ approved the final version of the manuscript. All authors have read and approved the final manuscript. JM and WY are the study guarantors.

Funding This study was supported by grants from the Key Research and Development Programs (2016YHC0900600, 2016YHC0900602); the Twelfth FiveYear Plan for Science and Technology Support (2012BAI03B03); Shanghai Health Commission of Health Industry Clinical Research Project (20194Y0209).

Competing interests None declared.

Patient and public involvement Patients and/or the public were not involved in the design, or conduct, or reporting or dissemination plans of this research.

Patient consent for publication All the subjects' parents provided signed informed consent.

Ethics approval The study was approved by the Research Ethics Committee of the Capital Institute of Paediatrics of China (2012062).

Provenance and peer review Not commissioned; externally peer reviewed.

Data availability statement Data are available upon reasonable request. Please contact Prof. Jie Mi.Email: jiemi12@vip.sina.com. Protocols and statistical analysis plans are required.

Open access This is an open access article distributed in accordance with the Creative Commons Attribution Non Commercial (CC BY-NC 4.0) license, which permits others to distribute, remix, adapt, build upon this work non-commercially, and license their derivative works on different terms, provided the original work is properly cited, appropriate credit is given, any changes made indicated, and the use is non-commercial. See: http://creativecommons.org/licenses/by-nc/4.0/.

ORCID iD

Weili Yan http://orcid.org/0000-0002-7633-7449

\section{REFERENCES}

1 WHO. Obesity and overweight, 2019. Available: https://www.who.int/ en/news-room/fact-sheets/detail/obesity-and-overweight

2 Skinner AC, Perrin EM, Moss LA, et al. Cardiometabolic risks and severity of obesity in children and young adults. $N$ Engl J Med 2015;373:1307-17.

3 Dehghan M, Akhtar-Danesh N, Merchant AT. Childhood obesity, prevalence and prevention. Nutr J 2005;4:24

4 Bauer KW, Marcus MD, El ghormli L, et al. Cardio-metabolic risk screening among adolescents: understanding the utility of body mass index, waist circumference and waist to height ratio. Pediatr Obes 2015;10:329-37.

5 Benmohammed K, Valensi P, Benlatreche M, et al. Anthropometric markers for detection of the metabolic syndrome in adolescents. Diabetes Metab 2015;41:138-44. 
6 Ma C-M, Yin F-Z, Liu X-L, et al. How to simplify the diagnostic criteria of metabolic syndrome in adolescents. Pediatr Neonatol 2017;58:178-84.

7 Zheng W, Zhao A, Xue Y, et al. Gender and urban-rural difference in anthropometric indices predicting dyslipidemia in Chinese primary school children: a cross-sectional study. Lipids Health Dis 2016;15:87.

8 Yan $\mathrm{W}$, Wang $\mathrm{X}$, Yao $\mathrm{H}$, et al. Waist-to-height ratio and $\mathrm{BMI}$ predict different cardiovascular risk factors in Chinese children. Diabetes Care 2006;29:2760-1.

9 Jiang Y, Dou Y-L, Xiong F, et al. Waist-to-height ratio remains an accurate and practical way of identifying cardiometabolic risks in children and adolescents. Acta Paediatr 2018:apa.14323

10 Browning LM, Hsieh SD, Ashwell M. A systematic review of waist-toheight ratio as a screening tool for the prediction of cardiovascular disease and diabetes: 0.5 could be a suitable global boundary value. Nutr Res Rev 2010;23:247-69.

11 Aguilar-Morales I, Colin-Ramirez E, Rivera-Mancía S, et al. Performance of Waist-To-Height ratio, waist circumference, and body mass index in discriminating Cardio-Metabolic risk factors in a sample of school-aged Mexican children. Nutrients 2018;10:nu10121850.

12 Ochoa Sangrador C, Ochoa-Brezmes J. Waist-to-height ratio as a risk marker for metabolic syndrome in childhood. A meta-analysis. Pediatr Obes 2018;13:421-32.

13 Tee JYH, Gan WY, Lim PY. Comparisons of body mass index, waist circumference, waist-to-height ratio and a body shape index (ABSI) in predicting high blood pressure among Malaysian adolescents: a cross-sectional study. BMJ Open 2020;10:e032874.

14 Yan Y, Liu J, Zhao X, et al. Regional adipose compartments confer different cardiometabolic risk in children and adolescents:: the China child and adolescent cardiovascular health study. Mayo Clin Proc 2019;94:1974-82.

15 Mi J, Wang TY, Meng LH, et al. Development of blood pressure reference standards for Chinese children and adolescents. Chinese Journal of Evidence Based Pediatric 2010;5:10.3969:97-102.

16 Association SCfCoCPs. Expert: control of hyperlipid in children and adolescents in China. China Tropical Medicine 2008;8:124-7.

17 American Diabetes Association. Diagnosis and classification of diabetes mellitus. Diabetes Care 2014;37 Suppl 1:S81-90.
18 Group of China Obesity Task Force. [Body mass index reference norm for screening overweight and obesity in Chinese children and adolescents]. Zhonghua Liu Xing Bing Xue Za Zhi 2004;25:97-102.

$19 \mathrm{Li} \mathrm{H}$, Ji C-Y, Zong X-N, et al. [Body mass index growth curves for Chinese children and adolescents aged 0 to 18 years]. Zhonghua $E r$ Ke Za Zhi 2009;47:493-8.

20 YQ W, Yang Y, Peng NN, et al. Waist circumference and waist circumference percentile curves for children and adolescents in Shanghai. J Sch Health 2012;33:1334-7.

21 Ashwell M, Gibson S. A proposal for a primary screening tool: 'Keep your waist circumference to less than half your height'. BMC Med 2014;12:207.

22 Zhao M, Bovet P, Ma C, et al. Performance of different adiposity measures for predicting cardiovascular risk in adolescents. Sci Rep 2017;7:43686.

23 Graves L, Garnett SP, Cowell CT, et al. Waist-to-height ratio and cardiometabolic risk factors in adolescence: findings from a prospective birth cohort. Pediatr Obes 2014:9:327-38.

24 Tompuri TT, Jääskeläinen J, Lindi V, et al. Adiposity criteria in assessing increased cardiometabolic risk in prepubertal children. Front Endocrinol 2019;10:410.

25 Seo J-Y, Kim JH. Validation of surrogate markers for metabolic syndrome and cardiometabolic risk factor clustering in children and adolescents: a nationwide population-based study. PLoS One 2017;12:e0186050.

26 Weili $\mathrm{Y}, \mathrm{He} \mathrm{B}$, Yao $\mathrm{H}$, et al. Waist-to-height ratio is an accurate and easier index for evaluating obesity in children and adolescents. Obesity 2007;15:748-52

27 Zhou D, Yang M, Yuan Z-P, et al. Waist-to-Height ratio: a simple, effective and practical screening tool for childhood obesity and metabolic syndrome. Prev Med 2014;67:35-40.

28 Expert Panel on Integrated Guidelines for Cardiovascular Health and Risk Reduction in Children and Adolescents, National Heart, Lung, and Blood Institute. Expert panel on integrated guidelines for cardiovascular health and risk reduction in children and adolescents: summary report. Pediatrics 2011;128 Suppl 5:peds.20092107C:S213-56.

29 Power C, Lake JK, Cole TJ. Measurement and long-term health risks of child and adolescent fatness. Int J Obes Relat Metab Disord 1997;21:507-26. 